\title{
Engineering an RNA-based tissue-specific platform for genetic editing through use of a miRNA-enabled Cas12a
}

\author{
Rasmus Møller, Kohei Oishi, and Benjamin R. tenOever \\ Icahn School of Medicine at Mount Sinai, New York, NY 10029
}

\section{ABSTRACT}

The capacity to edit genomes using CRISPR-Cas systems holds immense potential for countless genetic-based diseases. However, one significant impediment preventing broad therapeutic utilization is in vivo delivery. While genetic editing at a single cell level in vitro can be achieved with high efficiency, the capacity to utilize these same biologic tools in a desired tissue in vivo remains challenging. Non-integrating RNA virus-based vectors constitute efficient platforms for transgene expression and surpass several barriers to in vivo delivery. However, the broad tissue tropism of viral vectors raises the concern for off-target effects. Moreover, prolonged expression of the Cas proteins, regardless of delivery method, can accumulate aberrant RNAs leading to unwanted immunological responses. In an effort to circumvent these shortcomings, here we describe a versatile RNA virus-based technology that can achieve cellspecific activity and self-inactivation by combining host microRNA (miRNA) biology with the CRISPR-Cas12a RNA-guided nuclease. Exploiting the RNase activity of Cas12a, we generated a vector that self-inactivates upon delivery of Cas12a and an accompanying CRISPR RNA (crRNA). Furthermore, we show that maturation of the crRNA can be made dependent on cellspecific miRNAs, which confers cell-specificity. We demonstrate that this genetic editing circuit delivers diminished yet sufficient levels of Cas12a to achieve effective genome editing whilst inducing a minimal immunological response. It can also function in a cell-specific manner thereby facilitating in vivo editing and mitigating the risk of unwanted, off-target effects. 


\section{INTRODUCTION}

29 The CRISPR-Cas systems of archaea and many bacteria are sequence-specific adaptive defense systems that have evolved to cleave foreign nucleic acid ${ }^{1}$. This

31 defense system is dependent on acquisition and integration of foreign DNA

32 protospacers in a process generally referred to as adaptation ${ }^{2}$. Once integrated,

33 expression of the so-called protospacers generate a precursor CRISPR RNA (pre-

34 crRNA) which is further processed and matured to produce crRNA - comprised of a 5'

35 direct repeat and the spacer. Finally, crRNA is bound by a Cas nuclease to elicit

36 interference on incoming DNA as defined by complementarity of its guide RNA.

37 Moreover, as the protospacer DNA is inherited, adaptation of a single prokaryotic cell

38 can result in Lamarckian evolution for its offspring ${ }^{3}$.

While most of the Cas-nucleases only possess RNA-guided DNase activity, Cas12a

41 also has RNase function ${ }^{4}$. The RNase function is responsible for processing the pre-

42 crRNA by cleaving direct repeat sequences that flank the protospacer ${ }^{4}$. The crRNA that

43 is generated as a result of these processing events is sufficient for instilling specificity

44 onto the DNase activity of Cas12a. Similar to Cas9, Cas12a has also been repurposed

45 as a eukaryotic gene editor ${ }^{5-8}$. However, as Cas12a biology is still in its infancy, its

46 optimization lags behind that of Cas9. Despite this, the ability of Cas 12a to process its

47 own crRNA enables one to use it to generate the crRNA from diverse types of RNA so

48 long as it is flanked by direct repeats ${ }^{9}$. This activity not only allows one to generate

49 multiple crRNAs for any number of targets, but it has also enabled the generation of

50 mRNAs that both code for Cas12a and the desired guides on a single transcript ${ }^{10}$. This 
51 is in contrast to the most commonly applied Cas9 editing tool which demands a

52 separate DNA dependent RNA polymerase for production of Cas9 and the single guide

$53 \mathrm{RNA}^{6,11 .}$

Despite the immense potential of both the Cas 9 and Cas12a systems, one significant

56 impediment remains delivery of these large proteins alongside the desired crRNA(s).

57 This challenge is formidable, especially when one wishes to efficiently edit a large

58 number of cells to repair a genetic defect in vivo. This problem is further confounded by

59 the fact that maintaining Cas expression for longer periods of time can result in the

60 generation of off-target effects, aberrant RNAs, chromosomal translocations, and/or

61 removal of the Cas-expressing cells ${ }^{12,13}$. Given these challenges, the most optimal

62 genetic editors would be delivered with the efficiency of a virus in a manner that was

63 free of any possibility of genomic integration and would function only in a desired cell

64 type for the time required to achieve editing. To this end, we designed a self-

65 inactivating RNA that expresses Cas12a and processes its own crRNA in a cell-specific

66 manner that is amenable to RNA virus-based delivery.

68 Having some parallels with the CRISPR-Cas platform, host miRNAs are rooted in

69 defense systems albeit optimized for the targeting of RNA, rather than DNA ${ }^{14}$.

70 Generally described as RNA interference (RNAi), this defense system acquires double

71 stranded RNA (dsRNA) fragments from incoming virus and processes them into small

72 interfering RNAs (siRNAs) similar in length than that of crRNAs. Furthermore, like

73 crRNAs, siRNAs provide specificity to an otherwise non-specific nuclease. In the case 
74 of RNAi, siRNAs are bound by a family of Argonaute (AGO) proteins with the capacity to

75 cleave target mRNA in a sequence-specific manner ${ }^{15}$. The emergence of this system

76 was so evolutionarily successful, that the genes involved in this biology duplicated and

77 generated a similar small RNA based system commonly referred to as microRNAs

78 (miRNAs) ${ }^{16}$. Unlike the small RNA-based antiviral systems, miRNAs derive from the

79 host genome but are ultimately processed and utilized in a similar function, even

80 sharing some overlap with the processing machinery of the Type II CRISPR-Cas

81 system ${ }^{17}$. The general repurposing of this biology is believed to have happened

82 independently in plants and animals but, in both examples, the biogenesis of miRNA-

83 mediated regulation enabled another level of transcriptional control which coincided with

84 multicellularity ${ }^{16}$.

One significant difference between antiviral RNAi and host miRNA biology is the

87 complementarity between the small RNA and its target(s). In contrast to antiviral RNAi,

88 miRNAs do not generally bind with perfect complementarity to their targets and

89 therefore do not induce cleavage, owing to the fact that they are derived from host

90 genes $^{16}$. As a result, miRNAs are often considered 'fine-tuners' of host biology and are

91 thought to act over the course of days or weeks ${ }^{18}$. In contrast, virus-derived siRNAs

92 can engage their target with complete complementarity, as they derive from the

93 pathogen itself, and thus enable both cleavage and the recycling of the small RNA -

94 achieving silencing within hours ${ }^{16}$. However, as this difference in biology is almost

95 completely defined by complementarity, if one introduces a perfect binding site for a

96 given miRNA into a transcript, it will undergo potent silencing more akin to the normal 
97 activity of antiviral RNAi ${ }^{19}$. This exploitation of miRNA biology therefore enables one to

98 generate viruses or virus-based vectors which can be ubiquitously targeted or function

99 in a species-, tissue-, cell-, or even signaling-specifc manner based on endogenous

100 miRNA expression ${ }^{20-26}$.

101

102 In an effort to generate an RNA-based DNA editor that functions in a cell-specific

103 manner that would be amenable for in vivo use, we combined CRISPR-Cas and miRNA

104 biology. In brief, we utilize the fact that Cas12a processes its own pre-crRNA to make a 105 vector that delivers both Cas12a and crRNA and in doing so, inactivates itself. To this 106 end, we encode crRNAs in the 3'-UTR of Cas12a and show that it leads to self-

107 cleavage of its own transcript. Moreover, we demonstrate that delivery of this self108 inactivating construct is sufficient to achieve efficient gene editing. And lastly, we show 109 that processing of the pre-crRNA can be made to be dependent on miRNA-expression 110 thereby conferring cell-type specificity on our editing platform.

\section{RESULTS}

113 In an effort to build a single mRNA transcript that could both self-inactivate and function

114 in a cell-specific manner, we designed a construct encoding an enhanced green

115 fluorescent protein (GFP) and an HA epitope-tagged Cas12a separated by a P2A

116 peptide site $^{27}$ with its targeting instructions in the 3' UTR (Fig. 1a). To achieve self-

117 inactivation, we incorporated a crRNA in the 3' untranslated region (UTR) of the GFP-

118 Cas12a mRNA that consisted of a direct repeat and a spacer (DR). Accurate

119 processing of this crRNA relies on a second motif found 3' of the spacer comprising a 
120 perfect miRNA target (miR-T) site. In the presence of the cognate miRNA, Ago2 as part

121 of the RNA induced silencing complex (RISC), will be recruited and result in 3' cleavage

122 of the crRNA ${ }^{19}$. As miRNAs can be cell-specific, this synthetic construct would

123 inactivate itself ubiquitously while only generating functional crRNA in a desired cell type

124 where the cognate miRNA is present (Fig. 1a).

126 To initially characterize self-inactivation, we experimented with variations on the DR

127 design to ascertain the relationship between Cas12a engagement and loss of GFP

128 expression (Fig. 1b). To this end, we utilized either canonical direct repeats, direct

129 repeats that would be poorly or unable to be cleaved by Cas12a (A18G and UUAA,

130 respectively), or one in which the direct repeats were disrupted altogether (scrambled;

131 scrbl $)^{9,28}$. To determine how these constructs would function, they were introduced into

132 fibroblasts and monitored for GFP expression by both fluorescence microscopy and

133 western blot (Fig. 1b-c). These data demonstrated that the GFP expression from the

134 construct containing canonical direct repeats showed only low levels of fluorescence or

135 expression by western blot which could also be correlated with HA-Cas12a expression.

136 When the direct repeats were comprised of the A18G sites, fluorescence increased as

137 compared to canonical sites. This enhanced expression could also be further

138 corroborated by western blot analysis of both GFP and HA-Cas12a suggesting self-

139 inactivation was diminished with the $\mathrm{A} 18 \mathrm{G}$ sites. When the direct repeats were made to

140 be uncleavable by Cas12a (UUAA), GFP expression was comparable to a construct

141 lacking any direct repeats (scrbl). These data could be further validated by small RNA

142 northern blot which indicated that wild type direct repeats, and to a lesser extent A18G, 
143 was capable of generating a visible crRNA, inversely correlating to the GFP and HA-

144 Cas12a protein levels (Fig. 1c-d).

146 We next sought to make correct processing of the crRNA dependent on miRNA-

147 mediated cleavage to enable cell-specificity. To this end, we replaced the 3' direct

148 repeat with two target sites for an endogenously expressed miRNA, miR-106a or an

149 irrelevant control target sequence (ctrl-T) while keeping a wild-type direct repeat on the

$150 \quad 5$ ' end to mediate self-inactivation (Fig. 1b). GFP and Cas12a protein expression from

151 these two constructs were comparable to that containing two canonical direct repeats

152 indicating that a single direct repeat is sufficient for self-inactivation (Fig. 1b-c). In

153 contrast, the crRNA is no longer processed when the 3' direct repeat is replaced with

154 the control miRNA target sequence (ctrl-T) indicating a lack of cleavage (Fig. 1d).

155 However, when ctrl-T is replaced with target sites corresponding to miR-106a, which is 156 abundantly expressed in fibroblasts, we observe a distinct product corresponding to

157 Ago2-based cleavage (Fig. 1d). As Ago2 cleaves its target site between bases $10-11^{16}$,

158 the resulting crRNA contains an extended 3' terminus which should not impede Cas12a 159 targeting or specificity ${ }^{29}$.

161 To ascertain whether the product of 5' direct repeat and a 3' miRNA cleavage site

162 remains functional, we next expressed variants of our RNA construct that encoded a 163 crRNA targeting beta 2 microglobulin (B2M). In comparing transcripts lacking direct

164 repeats (scrbl), having both direct repeats, or containing a 5' direct repeat with either a 165 control 3' target sequence (ctrl) or miR-106a 3' sites we observe loss of MHC Class I, a 
proxy for B2M targeting, only in conditions in which the 3' end of the spacer contained a wild type direct repeat or the miR-106a target sites (Fig. 1d). These data demonstrate a $\sim 14 \%$ reduction of $\mathrm{MHC} 1$ with the canonical Cas12a targeting system which increases

169 to greater than $30 \%$ targeting in the presence of miR-106a despite the extended crRNA

170 (Fig. 1d-e). Together, these data suggest that miRNA biology can be exploited in

171 conjunction with Cas12a-based processing to generate a single RNA capable of both 172 self-inactivation and cell-specific targeting.

174 Given the capacity of a single transcript to both yield a functioning Cas 12 a editing 175 platform and undergo self-inactivation, we next assessed whether this biologic circuit 176 could be applied to a viral modality that would be most amenable to in vivo delivery. For 177 safety reasons ${ }^{30}$, this work was done with an RNA virus incapable of spread and 178 containing a scrambled crRNA with no complementarity to a genomic sequence. 179 Utilizing only the RNA dependent RNA polymerase (RdRp) of Nodamura virus and the 5' and 3' uncoding material required for RdRp recognition as described elsewhere ${ }^{31}$, we 181 generated a self-replicating RNA (herein referred to as a replicon) to express GFP, 182 Cas12a, and a 3' crRNA-containing UTR (Fig. 2a). As observed from our original 183 plasmid design, launching of this replicon showed self-inactivation was also achieved 184 using a viral-based delivery system (Fig. 2b). Comparable to what was observed using 185 DNA, the RNA-based circuit equivalent showed self-targeting with canonical direct 186 repeats (either one or two) with an intermediate phenotype observed for the A18G 187 variant. 
To determine whether the transcriptional response to our self-inactivating circuit would be amenable to in vivo use, we next performed bulk RNA sequencing to ascertain the transcriptional response to Cas12a expression and/or crRNA processing. To this end, we first compared the expression of Cas12a that was capable of self-inactivation and compared it to one incapable thereof. Surprisingly, this sequencing data set revealed

194 that in contrast to sustained expression of Cas12a alone, the self-inactivating plasmid resulted in a significant number of differentially expressed genes (DEGs) (Fig. 3a and Supplementary Table 1). All upregulated genes with a log2fold change greater than 1 and an adjusted p-value less than 0.01 were annotated as belonging to the interferon response (Supplementary Table 1). These data would indicated that Cas12a

199 processing of its own RNA results in a significant accumulation of aberrant RNA

200 capable of inducing the host antiviral defenses. In contrast, this same comparison using

201 the replicon-based platform yielded no DEGs (Fig. 3b). To determine if the lack of an 202 interferon signature in response to the replicon-based platform was simply the result of

203 having it generated in both conditions as a result of RdRp activity, we next compared 204 the plasmid-based Cas12a system with processable crRNA to the equivalent replicon 205 platform (Fig. 3c). This comparison yielded a larger number of DEGs but the interferon

206 signature remained limited to plasmid-based delivery of Cas $12 a$ and crRNA

207 demonstrating that the replicon self-inactivation is potent enough to prevent a cellular 208 antiviral response (Supplementary Table 2). This was further corroborated by replicon 209 read numbers which show that self-inactivation prevents any accumulation of either

210 positive or negative sense transcripts that might otherwise serve as pathogen 211 associated molecular patterns (Fig. 3d). 


\section{DISCUSSION}

214 Here we present data demonstrating that RNA-based platforms can be designed to

215 support safe and effective genetic editing. Based on the dual RNase and DNase

216 properties of Cas12a, we show that RNA constructs can be engineered to be self-

217 targeting. This attribute ensures that Cas12a and crRNA expression is temporal,

218 thereby minimizing off-target editing and the accumulation of aberrant and potentially

219 inflammatory RNA. In addition, self-inactivation also allows one to adopt a RNA virus-

220 based delivery system, as self-cleavage minimizes cytotoxicity and ensures no

221 persistence or genomic integration.

223 Having shown that an RNA-based replicon can deliver a self-inactivated Cas12a and

224 crRNA in the absence of a transcriptional response, we next sought to engineer this

225 construct so that it would only function in a desired cell type. In general, nucleic acid-

226 based therapeutics and gene therapy vectors rely on promoter elements that are

227 uniquely specific to a desired cell type. While this strategy has achieved some

228 noteworthy successes, use of DNA as a vector introduces other unwanted issues

229 including the need for entry into the nucleus and the possibility of genomic integration.

230 RNA-based vectors mitigate this risk by having no DNA phase and performing all of

231 their function in the cytoplasm ${ }^{32}$. Given these attributes, we chose to adapt miRNA-

232 based targeting as a means of instilling cell-specific activity. Here we show that the

233 addition of a perfect complementary miRNA target site can replace the 3' direct repeat

234 needed to liberate a desired crRNA and thus render its biology cell specific. Together 
235 with the knowledge that every tissue or cell-type has a unique miRNA profile, these data

236 suggest that one can engineer an RNA-based vector to efficiently enter the cytoplasm

237 and then function only in those cells where editing is desired.

239 The completion of the human genome project ushered in hope that treating genetic

240 diseases would soon become possible ${ }^{33}$. This dream was further fueled by the

241 discovery and repurposing of the bacterial and archaeal CRISPR-Cas immune systems

242 which provided unprecedented genome editing capabilities ${ }^{1}$. Indeed, recent efforts

243 have suggested that this promise is closer to reality than ever before as demonstrated

244 through the ex vivo editing of human T cells for therapeutic purposes ${ }^{13}$. While this

245 latter accomplishment may provide countless genetic treatments, the full potential of

246 CRISPR-based therapeutic still will require a vehicle for in vivo editing. Here we provide

247 a platform to enable this next advancement. By exploiting the biology of RNA viruses,

248 Cas12a, and miRNAs, here we demonstrate that one can design a single RNA that

249 could be systemically delivered with high efficiency but only function for a set amount of

250 time and only within a desired cell lineage. The basic principle underlying this biology

251 could be used with the replicon based strategy outlined here or with existing RNA virus

252 based genetic editors ${ }^{32}$. Taken together, with the rapid advancements of synthetic

253 biology, new CRISPR-Cas systems, and our understanding of virus-host interactions,

254 our progress towards in vivo editing may allow us to view genetic-based errors as

255 something that can be seamlessly overwritten. 


\section{METHODS}

259 Cells

260 All fibroblasts used in this paper were HEK293T cells which were maintained in Dulbecco's

261 Modified Eagle Medium (Gibcoß) supplemented with 1x penicillin-streptavidin solution

262 (Corning®) and 10\% fetal bovine serum (Corning®).

\section{Plasmids}

265 All constructs were synthesized by Twist Biosciences. The sequence will be deposited on NCBI 266 and the plasmid will be made available on AddGene following publication.

Western blot

269 Whole cell extract was prepared from live cells lysed in 1\% NP-40 lysis buffer. $2 \mu \mathrm{g}$ (pEGFP

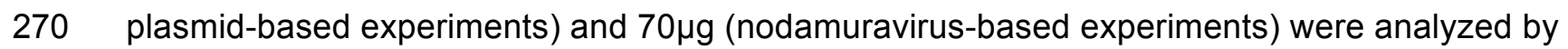

271 SDS-PAGE on a 4-15\% acrylamide gradient gel (BIO-RAD®) and gels were subsequently

272 blotted onto a $0.45 \mu \mathrm{m}$ nitrocellulose membrane (BIO-RAD®) and blocked in $5 \%$ milk in TBS for

$2731 \mathrm{~h}$ at room temperature. Blots were probed with the following primary antibodies in $5 \%$ milk in

274 TBS-T overnight at $4^{\circ} \mathrm{C}$ : anti-HA (clone HA-7, MilliporeSigma $\left.{ }^{\circledR}\right)$, anti-GFP (ab290, Abcam®),

275 anti-actin (clone Ab-5, Thermo Scientific $®$ ) and anti-GAPDH (G9545, MilliporeSigma ${ }^{\circledR}$ ). After 4

$276 \times 5$ min washes in $1 \times$ TBS-T, blots were probed with HRP-linked secondary antibody for $1 \mathrm{~h}$ at

277 room temperature (anti-mouse, NA931V or anti-rabbit, NA934V, GE Healthcare $囚$ ) and

278 developed using the Immobilon Western HRP Substrate Kit (MilliporeSigma®).

280 Small RNA Northern blot

281 Total RNA was extracted from live cells using TRIzol (Invitrogen). Northern blot was performed 282 as described in with $20 \mu \mathrm{g}$ total RNA per sample ${ }^{34}$. Probes included the following: B2M-crRNA 

developed on a Typhoon TRIO Storage Phosphorimager (GE®).

\section{Flow cytometry}

289 Roughly $7.5 \times 10^{5}$ cells/well were plated on 6-well plates. After attaching overnight, cells were 290 transfected using lipofectamine 2000 (Invitrogen $®$ ) and were passaged 1:5 when they reached $291 \sim 80 \%$ confluency for up to ten days. For flow cytometry analysis, cells were trypsinized and 292 washed 2 x stain buffer (FBS) (BD Biosciences ${ }^{\circledR}$ ) and stained using the BD Cytofix/Cytoperm 293 Fixation/Permeabilization Kit (BD Biosciences $\left.{ }^{\circledR}\right)$. The following antibodies and dyes were used: 294 anti-human HLA-A,B,C Pacific Blue (clone W6/32, BioLegend®), anti-HA Alexa Fluor 647 (clone 295 HA.11, BioLegend) and LIVE/DEAD stain Aqua (ThermoFisher ${ }^{\circledR}$ ). Fixed cells were analyzed on a 2019 Attune NxT Flow Cytometer. Data processing was done with FlowJo v. 10.6.2.

\section{RNA sequencing}

299 Total RNA was extracted using TRIzol (Invitrogen®). $1 \mu \mathrm{g}$ of total RNA per sample was used as

300 starting material for building Illumina libraries for deep sequencing. Libraries were built following 301 Illumina protocols using the TruSeq Stranded mRNA library prep kit (Illumina®). Libraries were 302 enriched for polyadenylated transcripts and sequenced on an Illumina NextSeq 500. Alignment 303 of reads was done using STAR alignment to the human reference genome (hg38) and

304 differential gene expression and statistics were determined by the DEseq2 protocol. Reads 305 mapping to the Nodamuravirus replicon were aligned using Bowtie2. 
bioRxiv preprint doi: https://doi.org/10.1101/2020 03 04.977363: this version posted March 5,2020 . The copyright holder for this preprin (which was not certified by peer review) is the author/funder, who has granted bioRxiv a license to display the preprint in perpetuity. It is made available under aCC-BY-ND 4.0 International license.

\section{FIGURES}

a
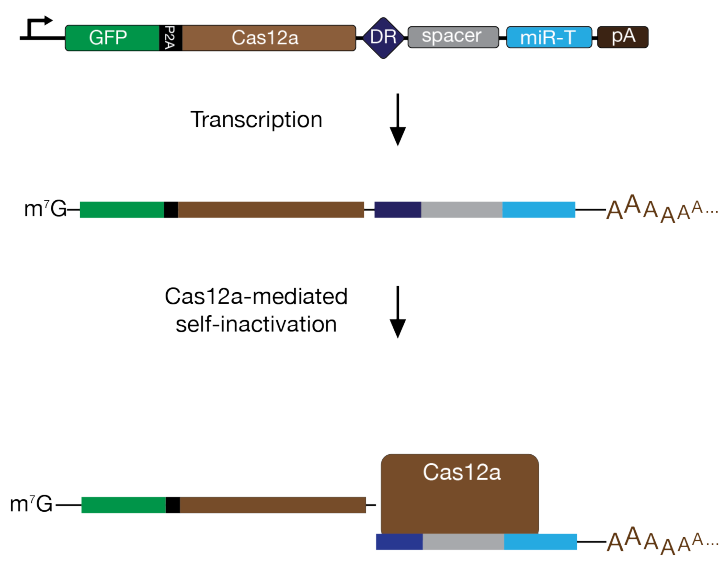

Vector inactivation
in absence of miRNA $\quad \begin{gathered}\text { Ago2-mediated cleavage } \\ \text { in presence of miRNA }\end{gathered}$

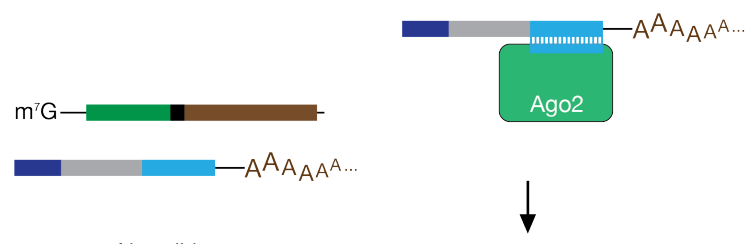

No editing

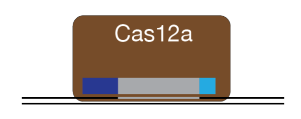

b

Gene editing

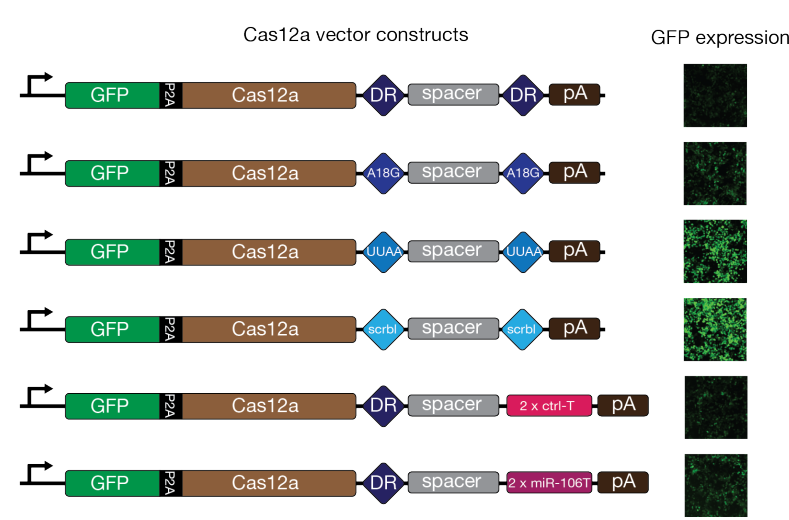

C

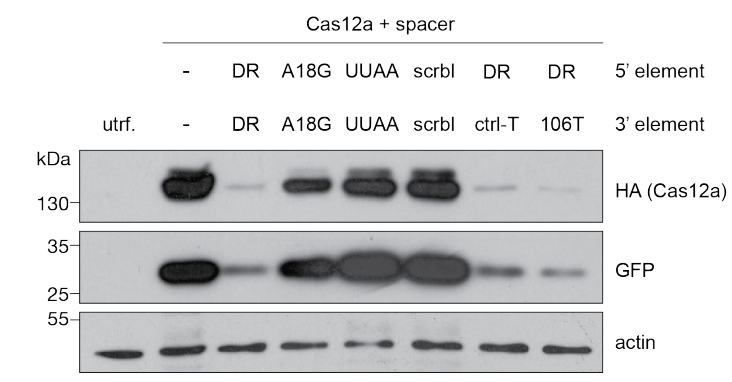

d

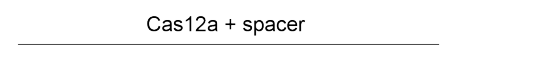

DR A18G UUAA scrbl DR DR 5' element
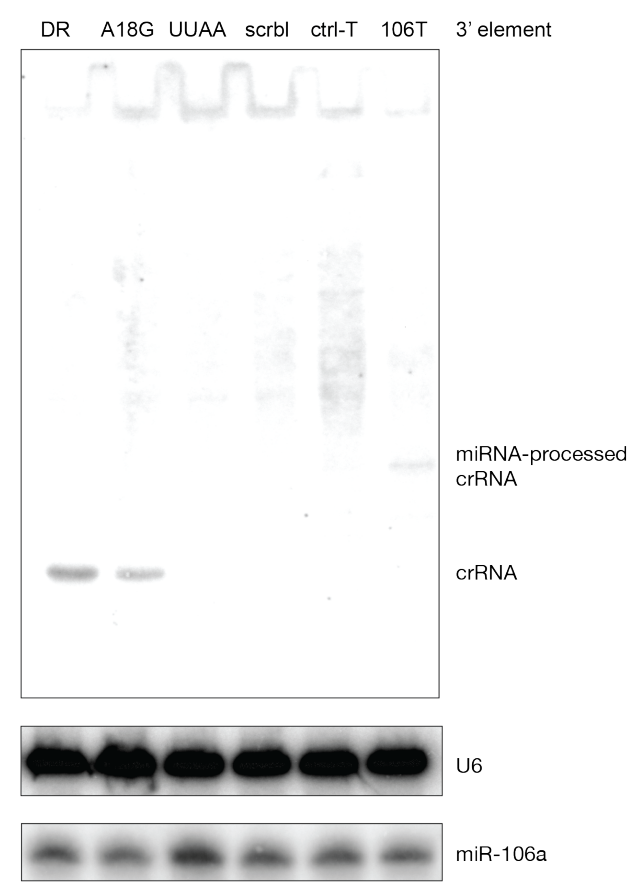

e

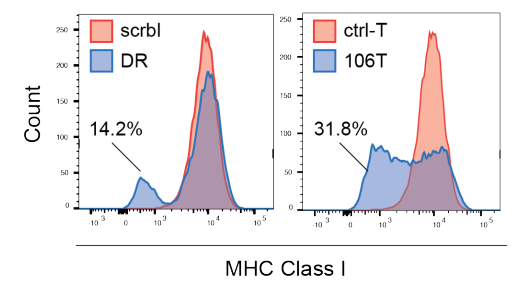




\section{Fig. 1: Self-inactivation and tissue specificity of Cas12a vector.}

313 a, A schematic of how self-inactivation and miRNA-based tissue specificity is incorporated into

314 one single Cas12a vector. GFP and Cas12a are encoded in one open reading frame separated

315 by a P2A site. The 3'-UTR of the same transcript consists of a direct repeat (DR), a spacer with

316 complementarity to a genomic target and a sequence with perfect complementarity to a miRNA

317 referred to as a miRNA-target site (miR-T) and lastly an SV40 polyadenylation signal (pA). b,

318 Variations on the construct depicted in a with a mutation of nucleotide 18 in the direct repeat

319 from an $A$ to a $G(A 18 G)$, inversion of nucleotides 16-19 from AAUU to UUAA (UUAA) or a

320 scrambled sequence of the entire direct repeat (scrbl). The last two constructs have two target

321 sites to either a control-miRNA (ctrl-miRT) or miR-106a (miR-106T). Fluorescence images are

322 representative of GFP expression 48 hours after transfection with the constructs indicated. c,

323 Western blot of cells transfected for 48 hours with the constructs depicted in $\mathbf{b},-$, no crRNA in

324 3'-UTR, utrf., untransfected cells. d, Small RNA Northern blot of total RNA from cells as in c,

325 U6, U6 snRNA (loading control). e, MHC Class I cell surface expression measured ten days by

326 flow cytometry ten days prost transfection. Data from cells transfected with the constructs

327 overlaid as indicated. 
a

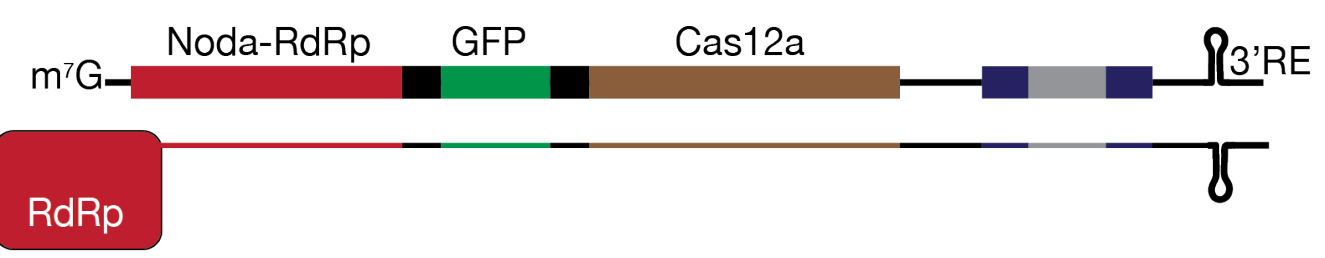

\section{$\uparrow$ self-amplification}
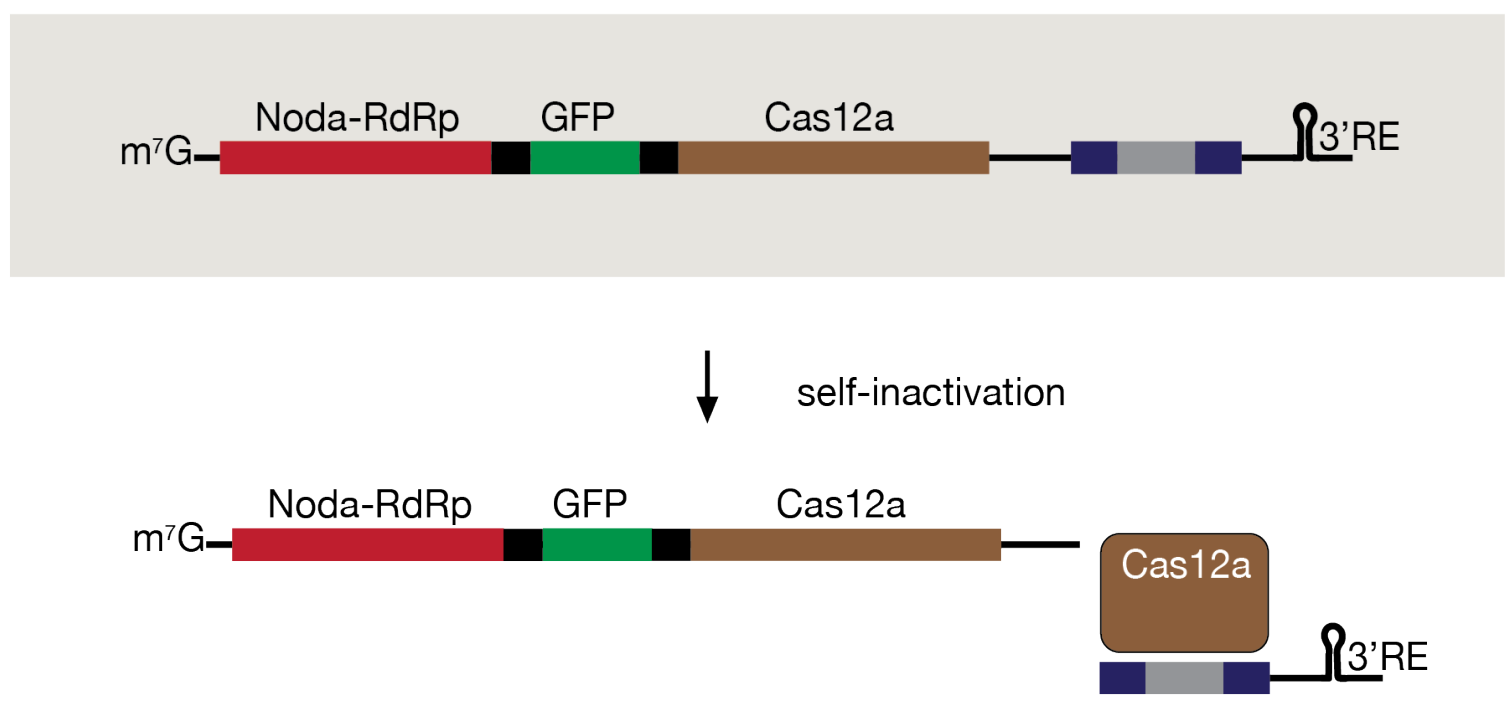

b 
bioRxiv preprint doi: https://doi.org/10.1101/2020 03.04.977363; this version posted March 5, 2020. The copyright holder for this preprint (which was not certified by peer review) is the author/funder, who has granted bioRxiv a license to display the preprint in perpetuity. It is made available under aCC-BY-ND 4.0 International license.

335 Fig. 2: Delivery of Cas12a from a self-inactivating replicon.

336 a, Schematic of a self-inactivating replicon construct. Nodamuravirus RNA dependent RNA

337 polymerase (Noda-RdRp), GFP and Cas12a are encoded in one open reading frame separated

338 by P2A sites. Downstream of the open reading frame, a spacer (grey) flanked by two direct

339 repeats (dark blue) are inserted. $\mathbf{b}$, Western blot of cells transfected with the replicon constructs

340 indicated (as in Fig. 1c). 
a

\section{Plasmid vector}

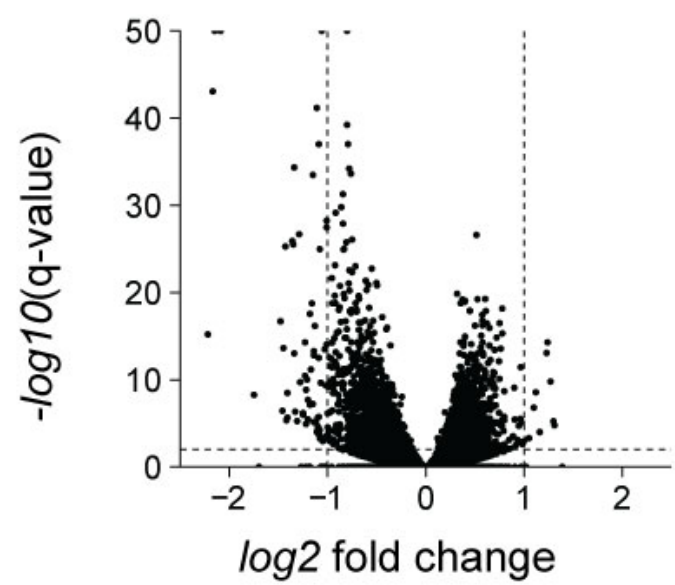

C

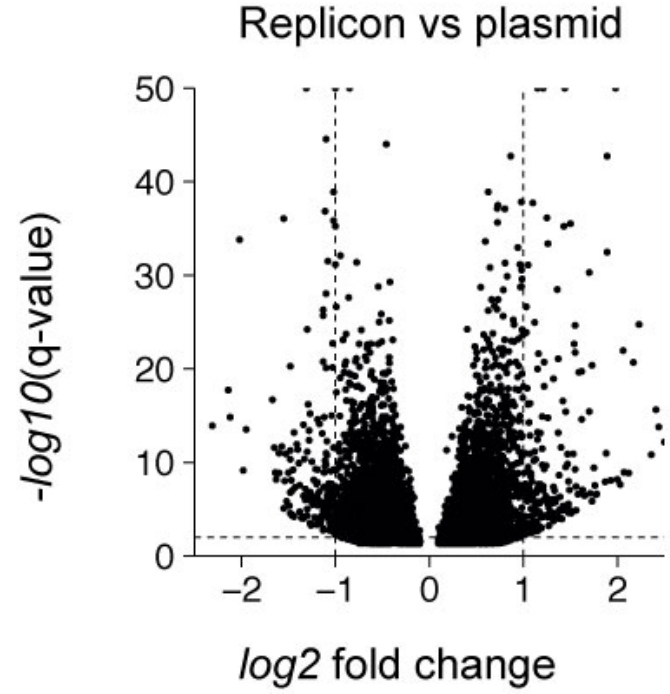

b Replicon vector

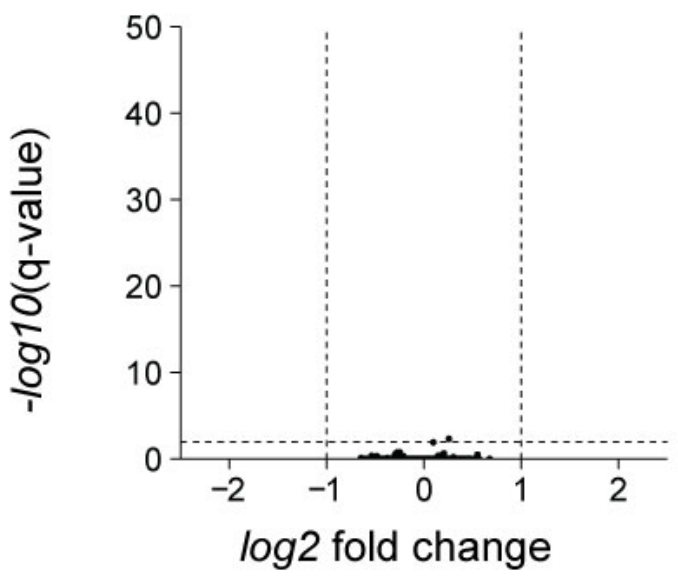

d

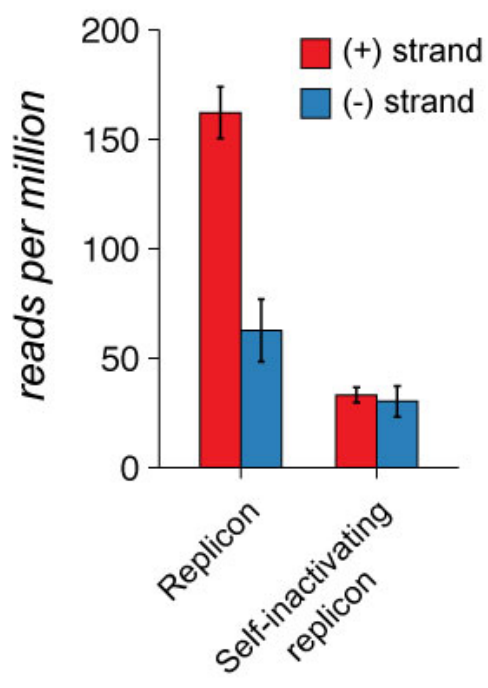


350 Fig. 3: Transcriptional response to self-inactivating Cas12a vectors.

351 a, Plot depicting differential gene expression of host genes in cells transfected with a plasmid-

352 based Cas12a construct containing direct repeats in the 3'-UTR compared to cells transfected

353 with a comparable construct without direct repeats. Each dot represents a gene plotted by its

$354 \log 2$ fold change between the two conditions and - $\log 10$ of the adjusted $p$-value (q) determined

355 based on triplicate samples. Horizontal line marks a q-value $=0.01$ and Vertical lines mark a

$356 \log 2$ fold change of -1 and $1 . \mathbf{b}$, same as $\mathbf{a}$, but comparing replicon-based Cas 12 a construct

357 containing direct repeats against a comparable construct without direct repeats. c, same as a,

358 but comparing plasmid-based Cas12a with direct repeats to replicon-based Cas12a with direct

359 repeats. d, Stranded read numbers aligning to the replicon as number of reads per million of

360 total reads. Error bars represent standard deviation from three replicates. 
SUPPLEMENTARY MATERIAL

375

376 Table 1. Differentially expressed genes in response to self-inactivating vs. non-

377 processable plasmid-based Cas12a vector. The control group are fibroblasts expressing 378 GFP, Cas12a, and a crRNA lacking direct repeats performed in triplicate. The experimental 379 group are fibroblasts expressing GFP, Cas12a, and a crRNA flanked with direct repeats, also

380 performed in triplicate. Total reads per sample are given. Shown are gene names, mean count 381 per gene, log2fold change in comparing triplicate samples in deSeq2, Standard error and 382 adjusted p-value (q value). Raw data to be deposited on NCBI GEO.

Table 2. Differentially expressed genes in response to self-inactivating plasmid-based

Cas12a versus the corresponding replicon-based construct. The control group are

fibroblasts expressing GFP, Cas12a, and a crRNA flanked with direct repeats performed in triplicate. The experimental group are fibroblasts expressing GFP, Cas12a, and a crRNA flanked with direct repeats as delivered by RNA replicon, also performed in triplicate. Total reads per sample are given. Shown are gene names, mean count per gene, log2fold change in comparing triplicate samples in deSeq2, Standard error and adjusted $p$-value (q value). Raw

391 data to be deposited on NCBI GEO. 


\section{REFERENCES}

3991 Marraffini, L. A. \& Sontheimer, E. J. CRISPR interference: RNA-directed adaptive

$400 \quad$ immunity in bacteria and archaea. Nat Rev Genet 11, 181-190, doi:10.1038/nrg2749

$401 \quad$ (2010).

4022 Makarova, K. S. et al. An updated evolutionary classification of CRISPR-Cas systems.

$403 \quad$ Nat Rev Microbiol 13, 722-736, doi:10.1038/nrmicro3569 (2015).

4043 van der Oost, J., Jore, M. M., Westra, E. R., Lundgren, M. \& Brouns, S. J. CRISPR-

405

based adaptive and heritable immunity in prokaryotes. Trends Biochem Sci 34, 401-407,

406 doi:10.1016/j.tibs.2009.05.002 (2009).

4074 Fonfara, I., Richter, H., Bratovic, M., Le Rhun, A. \& Charpentier, E. The CRISPR-

408 associated DNA-cleaving enzyme Cpf1 also processes precursor CRISPR RNA. Nature 532, 517-521, doi:10.1038/nature17945 (2016).

4105 Cho, S. W., Kim, S., Kim, J. M. \& Kim, J. S. Targeted genome engineering in human 411 cells with the Cas9 RNA-guided endonuclease. Nat Biotechnol 31, 230-232, 412 doi:10.1038/nbt.2507 (2013).

4136 Cong, L. et al. Multiplex genome engineering using CRISPR/Cas systems. Science 339, $414 \quad$ 819-823, doi:10.1126/science.1231143 (2013).

4157 Hwang, W. Y. et al. Efficient genome editing in zebrafish using a CRISPR-Cas system. $416 \quad$ Nat Biotechnol 31, 227-229, doi:10.1038/nbt.2501 (2013).

4178 Mali, P. et al. RNA-guided human genome engineering via Cas9. Science 339, 823-826, 418 doi:10.1126/science.1232033 (2013).

4199 Zetsche, B. et al. Multiplex gene editing by CRISPR-Cpf1 using a single crRNA array. Nat Biotechnol 35, 31-34, doi:10.1038/nbt.3737 (2017).

42110 Campa, C. C., Weisbach, N. R., Santinha, A. J., Incarnato, D. \& Platt, R. J. Multiplexed genome engineering by Cas12a and CRISPR arrays encoded on single transcripts. Nat Methods 16, 887-893, doi:10.1038/s41592-019-0508-6 (2019). 
42411 Jinek, M. et al. A programmable dual-RNA-guided DNA endonuclease in adaptive

425 bacterial immunity. Science 337, 816-821, doi:10.1126/science.1225829 (2012).

42612 Koo, T., Lee, J. \& Kim, J. S. Measuring and Reducing Off-Target Activities of

427 Programmable Nucleases Including CRISPR-Cas9. Mol Cells 38, 475-481,

428 doi:10.14348/molcells.2015.0103 (2015).

42913 Stadtmauer, E. A. et al. CRISPR-engineered T cells in patients with refractory cancer.

$430 \quad$ Science 367, doi:10.1126/science.aba7365 (2020).

43114 tenOever, B. R. The Evolution of Antiviral Defense Systems. Cell Host Microbe 19, 142-

$432 \quad$ 149, doi:10.1016/j.chom.2016.01.006 (2016).

43315 Mello, C. C. \& Conte, D., Jr. Revealing the world of RNA interference. Nature 431, 338-

$434 \quad 342$, doi:10.1038/nature02872 (2004).

43516 Bartel, D. P. Metazoan MicroRNAs. Cell 173, 20-51, doi:10.1016/j.cell.2018.03.006

$436 \quad$ (2018).

$43717 \quad$ Aguado, L. C. et al. RNase III nucleases from diverse kingdoms serve as antiviral

$438 \quad$ effectors. Nature 547, 114-117, doi:10.1038/nature22990 (2017).

43918 Aguado, L. C. et al. microRNA Function Is Limited to Cytokine Control in the Acute

440 Response to Virus Infection. Cell Host Microbe 18, 714-722,

$441 \quad$ doi:10.1016/j.chom.2015.11.003 (2015).

44219 tenOever, B. R. RNA viruses and the host microRNA machinery. Nat Rev Microbiol 11,

443 169-180, doi:10.1038/nrmicro2971 (2013).

44420 Brown, B. D., Venneri, M. A., Zingale, A., Sergi Sergi, L. \& Naldini, L. Endogenous

445 microRNA regulation suppresses transgene expression in hematopoietic lineages and

446 enables stable gene transfer. Nat Med 12, 585-591, doi:10.1038/nm1398 (2006).

$44721 \quad$ Perez, J. T. et al. MicroRNA-mediated species-specific attenuation of influenza A virus.

$448 \quad$ Nat Biotechnol 27, 572-576, doi:10.1038/nbt.1542 (2009). 
44922 Langlois, R. A. et al. MicroRNA-based strategy to mitigate the risk of gain-of-function

$450 \quad$ influenza studies. Nat Biotechnol 31, 844-847, doi:10.1038/nbt.2666 (2013).

45123 Langlois, R. A., Varble, A., Chua, M. A., Garcia-Sastre, A. \& tenOever, B. R.

452 Hematopoietic-specific targeting of influenza A virus reveals replication requirements for 453 induction of antiviral immune responses. Proc Natl Acad Sci U S A 109, 12117-12122, 454 doi:10.1073/pnas.1206039109 (2012).

45524 Pham, A. M., Langlois, R. A. \& TenOever, B. R. Replication in cells of hematopoietic $456 \quad$ origin is necessary for Dengue virus dissemination. PLoS Pathog 8, e1002465, 457 doi:10.1371/journal.ppat.1002465 (2012).

45825 Moller, R. et al. miRNA-mediated targeting of human cytomegalovirus reveals biological 459 host and viral targets of IE2. Proc Natl Acad Sci U S A 115, 1069-1074, 460 doi:10.1073/pnas.1719036115 (2018).

46126 Aguado, L. C. et al. Homologous recombination is an intrinsic defense against antiviral RNA interference. Proc Natl Acad Sci U S A 115, E9211-E9219, doi:10.1073/pnas.1810229115 (2018).

46427 Sharma, P. et al. 2A peptides provide distinct solutions to driving stop-carry on translational recoding. Nucleic Acids Res 40, 3143-3151, doi:10.1093/nar/gkr1176 (2012). RNAs from mRNA transcripts in mammalian cells. Nat Chem Biol 13, 839-841,

47029 Strohkendl, I., Saifuddin, F. A., Rybarski, J. R., Finkelstein, I. J. \& Russell, R. Kinetic 471 Basis for DNA Target Specificity of CRISPR-Cas12a. Mol Cell 71, 816-824 e813, 472 doi:10.1016/j.molcel.2018.06.043 (2018).

$473 \quad 30 \quad$ Baltimore, D. et al. Biotechnology. A prudent path forward for genomic engineering and 474 germline gene modification. Science 348, 36-38, doi:10.1126/science.aab1028 (2015). 
47531 Gitlin, L., Hagai, T., LaBarbera, A., Solovey, M. \& Andino, R. Rapid evolution of virus

476 sequences in intrinsically disordered protein regions. PLoS Pathog 10, e1004529,

477 doi:10.1371/journal.ppat.1004529 (2014).

47832 Park, A. et al. Sendai virus, an RNA virus with no risk of genomic integration, delivers

479 CRISPR/Cas9 for efficient gene editing. Mol Ther Methods Clin Dev 3, 16057,

480 doi:10.1038/mtm.2016.57 (2016).

48133 Collins, F. S., Morgan, M. \& Patrinos, A. The Human Genome Project: lessons from

482 large-scale biology. Science 300, 286-290, doi:10.1126/science.1084564 (2003).

48334 Pall, G. S. \& Hamilton, A. J. Improved northern blot method for enhanced detection of

484 small RNA. Nat Protoc 3, 1077-1084, doi:10.1038/nprot.2008.67 (2008). 